\title{
Unhappy or unsatisfied: distinguishing the role of negative affect and need frustration in depressive symptoms over the academic year and during the COVID-19 pandemic
}

\author{
Shelby L. Levine ${ }^{1}$ (D) . Claire J. Brabander ${ }^{1} \cdot$ Amanda M. Moore $^{1} \cdot$ Anne C. Holding $^{1} \cdot$ Richard Koestner $^{1}$
}

Accepted: 27 November 2021 / Published online: 1 December 2021

○ The Author(s), under exclusive licence to Springer Science+Business Media, LLC, part of Springer Nature 2021

\begin{abstract}
Mental health problems are becoming increasingly prevalent across college campuses. Past research has found that negative affect and frustration of basic psychological needs contribute to the development of depressive symptoms, but there is limited research which compares whether these are antecedents or concomitants of depressive symptoms. The present set of studies aimed to distinguish the differential associations of affect and need frustration on depressive symptoms. Students $\left(\mathrm{N}_{\text {study } 1}=379 ; \mathrm{N}_{\text {study } 2}=235\right)$ completed measures on negative affect, need frustration (e.g., relatedness, competence, and autonomy), and depressive symptoms over an academic year and during the start of the COVID-19 pandemic. In both samples, fully cross-lagged path models were used to examine the relation between need frustration, negative affect, and depressive symptoms over time. Across both studies, basic psychological need frustration was the only consistent predictor of both negative affect and depressive symptoms over time, suggesting that need frustration is an antecedent of depressive symptoms over time, and especially during vulnerable time periods. Additionally, in Study 2, reports from close others confirm that need frustration is the largest indicator of depressive presentation in students. These results highlight the relative importance of basic psychological need frustration in predicting depressive symptoms in university students.
\end{abstract}

Keywords Self-determination theory $\cdot$ Basic psychological needs $\cdot$ Affect $\cdot$ Depressive symptoms

\section{Introduction}

Depression is one of the most common and persistent mental health problems that university students report (Song et al., 2008). Depressive symptoms can persist throughout university and can significantly affect students' academics, relationships, and daily functioning (Porter, 2018). The increased prevalence of mental health problems has spurred researchers to explore what contributes to ill-being on university campuses. Previous research has shown that both negative affect and frustration of basic psychological needs (e.g. autonomy, competence, and relatedness) can contribute to depressive symptoms (Vansteenkiste \& Ryan, 2013; Watson et al., 1988). Experiencing increased negative affect is closely linked to experiencing depressive symptoms (Watson

Shelby L. Levine

Shelby.levine@mail.mcgill.ca

1 McGill University, $2001 \mathrm{McGill}$ College, Montreal, QC H3A 1G1, Canada et al., 1988). Similarly, frustration of one's basic psychological needs, thwarting of a sense of competence, relatedness, or autonomy, is associated with increased likelihood of suffering from depressive symptoms (Costa et al., 2015; Ryan $\&$ Deci, 2017).

However, the conceptual overlap of negative affect, need frustration, and depressive symptoms has yet to be explored, even though these constructs are often highly correlated. The present study aimed to examine how need frustration, negative affect and depressive symptoms develop over the course of an academic year and during the COVID-19 pandemic. The main goal of this research was to be able to better understand what might put students at risk of experiencing more severe depressive symptoms and ill-being over time.

\section{Depressive symptoms}

Depressive symptoms, along with other mental health problems, have become increasingly prevalent across university campuses over recent decades (Porter, 2018; Rosenthal \& Schreiner, 2000; Storrie et al., 2010). Reports 
of depressive symptoms among university students are higher than in the adult population and higher than that of their peers who do not attend university (Chen et al., 2013; Wells et al., 1987). Additionally, throughout the duration of university, depressive symptoms persist; one study found that $60 \%$ of the students who indicated a mental health problem at the start of a study reported a mental health problem again 2 years later (Zivin et al., 2009). The prevalence and persistence of depressive symptoms compromises the daily functioning of students and makes it difficult for them to pursue their academic career (Song et al., 2008).

Depressive symptoms commonly present as heightened negative affect, anhedonia (loss of positive affect), weight loss or gain, motor retardation, impaired concentration, insomnia, restlessness, agitation, and feelings of worthlessness (Trivedi, 2004). On average, students report increased depressive symptoms over an academic year (Levine, Milyavskaya, et al., 2020). If antecedents can be identified, students could receive early support to counteract more mild depressive symptoms (Jackson et al., 2003). The current research aims to distinguish whether negative affect and need frustration are distinct antecedents of depressive symptoms across the academic year.

\section{Affect}

Affect is a critical component of psychopathology, and often a core symptom of mood and anxiety disorders. Affect is a general measure of a person's emotional experience over time (Emmons, 1992). Affect integrates one's physiological and cognitive appraisals, as well as the valence and intensity of an experience (Munezero, et al., 2014). To determine an individual's well and ill-being, researchers will often measure general affect (Emmons, 1992; Watson \& Clark, 1995).

Negative affect is a strong predictor and correlate of psychopathology generally (Watson \& Clark, 1995). Additionally, high levels of negative affect are highly correlated to symptoms and diagnoses of depression (Nima et al., 2013; Watson et al., 1988). Affect can also reinforce cognitive patterns, for example, ruminating about negative feelings can often enhance depressive symptoms, like feelings of worthlessness, insomnia or inattention (Raes et al., 2012). High negative affect is often observed prior to the onset of depression and may actively contribute to the development and worsening of depressive symptoms (Watson et al., 1988). The current research examines how negative affect relates to need frustration and depressive symptoms during emerging adulthood.

\section{Basic psychological needs theory}

Basic psychological needs theory, a mini-theory within Self Determination Theory (Ryan \& Deci, 2017), posits that need frustration contributes to ill-being (Vansteenkiste et al., 2020). A person's three basic psychological needs are competence (feeling able and confident in one's abilities), relatedness (feeling close to others), and autonomy (feeling free and volitional) (Ryan \& Deci, 2017). These needs must be satisfied in order to promote one's growth, vitality, and general psychological well-being, while active need thwarting leads to frustration, dissatisfaction, and ill-being (Diener \& Oishi, 2000). There are many aspects of university life that may be need frustrating for an individual, for example receiving grades below one's expectations (competence frustration), feeling isolated from one's peers (relatedness frustration), or perhaps feeling compelled to follow a certain career path (autonomy frustration). The current research aims to explore whether need frustration serves as a predictor of depressive symptoms over the academic year.

Need frustration is the active thwarting of one's needs, and need dissatisfaction is the lack of fulfillment of one's basic needs (Ryan \& Deci, 2017). For example, a plant will not fair well if it does not have sunlight (low need satisfaction) but will wither and die quickly if you use salt water on it (need frustration). When controlling for need frustration, need dissatisfaction did not predict ill-being, meaning need frustration is a unique contributor to negative outcomes (Cordeiro et al., 2016). For example, an environment where one does not get along with colleagues will result in low feelings of relatedness and low well-being, but a workplace where one is rejected and socially isolated by others effectively thwarts relatedness and leads to ill-being (Schultz et al., 2015; Vansteenkiste \& Ryan, 2013).

Need frustration often mediates the relationship between negative environments and depressive symptoms, suggesting that psychological needs may play a role in explaining why psychopathology occurs (Campbell et al., 2018). However, there have been only two studies that examined the relation between need frustration and ill-being in multi-wave longitudinal studies (Cordeiro et al., 2016; Vermote et al., 2021). Self-determination theory suggests that need frustration is a unique predictor of ill-being, but without rigorous longitudinal research, how can we determine whether need frustration precedes ill-being, or whether it is just a natural correlate of ill-being over time.

\section{Study 1}

The current research uses fully cross-lagged path modelling to examine the associations between need frustration, 
negative affect, and depressive symptoms over the academic year. Conceptually, there is a lot of overlap between these constructs, and the current methodology allows us to observe how these constructs influence each other over time while controlling for that shared variance. We hypothesized that both negative affect and need frustration would be unique predictors of depressive symptoms over the academic year, and that these variables would have reciprocal accumulation. In order to test these hypotheses, we conducted a 3-wave longitudinal survey over the course of a full 8-month academic year at a large Canadian university, and focused on students' reports of affect, basic psychological needs and depressive symptoms at beginning (September), middle (December) and end of the academic year (April). This study aims to highlight how selfdetermination theory can help our understanding of how depressive symptoms develop in university students, by highlighting the incremental validity of basic psychological need frustration relative to negative affect.

\section{Method}

\section{Participants and procedure}

The 379 participants for this year-long online study were recruited from a large Canadian university. Participants were undergraduate and graduate university students $\left(\mathrm{M}_{\mathrm{age}}=20.44, \mathrm{SD}=3.19\right)$ of which $85 \%$ were female, and $89 \%$ were undergraduate students. The ethnic background of our sample was predominantly European descent (52\%) but included 33\% Asian descent, 2\% African descent, 7\% Middle Eastern descent, 5\% Hispanic, and 1\% First Nations. Recruitment was done through traditional and online platforms such as posters around campus, lecture hall announcements, and Facebook posts. The study was conducted through a series of online surveys administered through Qualtrics at 3 time points throughout the school year in September (T1), December (T2) and April (T3). The surveys assessed psychological needs, affect, and depressive symptoms. The current study examined the data that was collected in the 2018-2019 academic year, for which $80 \%$ of participants were retained for all surveys ${ }^{1}(\mathrm{~T} 1: \mathrm{N}=379$, T2: $N=333$, T3: $N=304)$. Participants were compensated up to $\$ 50$ for their time.

\footnotetext{
1 There were no significant differences between individuals who dropped out of the study on all baseline and mid-year measurements. A summary of this analysis is included on OSF: https://osf.io/a6feq/.
}

\section{Measures}

\section{Affect}

The Scale of Positive and Negative Experience (SPANE) was used to assess negative affect (Emmons, 1992). Emmons' (1992) scale reliably and consistently measures negative affect. Five adjectives, such as unhappy and frustrated, are used to describe negative affect. Participants rated these items on a 7-point Likert scale ranging from 1 "I felt it not at all" to 7 "I felt it extremely much". The ratings were averaged to yield a negative affect score $\left(\alpha_{\text {NAT1 }}=0.75\right.$, $\left.\alpha_{\text {NAT2 }}=0.84, \alpha_{\text {NAT3 }}=0.83\right)$.

\section{Basic psychological need frustration}

The 9-item Balanced Measure of Psychological Needs scale (BMPN) was used to assess psychological need frustration (Cordeiro et al., 2016; Sheldon \& Hilpert, 2012). This scale uses three statements each to assess need frustration for each basic psychological need (relatedness, competence, and autonomy). An example of a prompt to measure relatedness frustration was "I feel lonely"; a prompt to measure competence frustration was "I experienced some kind of failure or was unable to do well at something"; and to measure autonomy frustration, "I feel pressured in my life". Participants rated their agreement with a series of statements on a 7-point Likert scale ranging from 1 "not at all true" to 7 "very true". All the frustration items were averaged to compute a measure of overall psychological need frustration $\left(\alpha_{\mathrm{NFT} 1}=0.77, \alpha_{\mathrm{NFT} 2}=0.75, \alpha_{\mathrm{NFT} 3}=0.86\right)$.

\section{Depressive symptoms}

The Centre for Epidemiologic Studies Depression Scale Revised (CESD-R 10) was used to assess symptoms of depression (Andresen et al., 1994). The CESD-R 10 is a validated and reliable self-report measure of depression symptoms that focuses on the affectivity component of depressed mood (Andresen et al., 1994). The scale includes items such as "I could not get going" and "I was bothered by things that usually don't bother me". It is measured on a four-point Likert scale ranging from 1 "rarely or none of the time ( $<1$ day)" to 4 "most or all the time (5-7 days)". A depressive symptoms score was computed by averaging the ten items $\left(\alpha_{\mathrm{T} 1}=0.79, \alpha_{\mathrm{T} 2}=0.85, \alpha_{\mathrm{T} 3}=0.86\right)$. 
Table 1 Mean, standard deviations and correlations between all variables of interest

\begin{tabular}{llllllllll}
\hline Variable & Mean(SD) & 2 & 3 & 4 & 5 & 6 & 7 & 8 & 9 \\
\hline 1. T1 Dep & $2.06(0.51)$ & $\mathbf{0 . 7 4}$ & $\mathbf{0 . 5 6}$ & $\mathbf{0 . 5 2}$ & $\mathbf{0 . 4 4}$ & $\mathbf{0 . 3 8}$ & $\mathbf{0 . 4 0}$ & $\mathbf{0 . 3 2}$ & $\mathbf{0 . 3 0}$ \\
2. T1 NA & $3.50(1.08)$ & 1 & $\mathbf{0 . 5 4}$ & $\mathbf{0 . 4 0}$ & $\mathbf{0 . 4 3}$ & $\mathbf{0 . 3 0}$ & $\mathbf{0 . 3 0}$ & $\mathbf{0 . 2 8}$ & $\mathbf{0 . 2 6}$ \\
3. T1 NF & $3.64(1.10)$ & - & 1 & $\mathbf{0 . 3 9}$ & $\mathbf{0 . 3 9}$ & $\mathbf{0 . 5 0}$ & $\mathbf{0 . 2 8}$ & $\mathbf{0 . 2 7}$ & $\mathbf{0 . 4 0}$ \\
4. T2 Dep & $2.29(0.61)$ & - & - & 1 & $\mathbf{0 . 7 8}$ & $\mathbf{0 . 6 7}$ & $\mathbf{0 . 4 4}$ & $\mathbf{0 . 3 6}$ & $\mathbf{0 . 3 5}$ \\
5. T2 NA & $3.95(1.28)$ & - & - & - & 1 & $\mathbf{0 . 6 7}$ & $\mathbf{0 . 4 1}$ & $\mathbf{0 . 4 2}$ & $\mathbf{0 . 3 8}$ \\
6. T2 NF & $4.02(1.25)$ & - & - & - & - & 1 & $\mathbf{0 . 4 0}$ & $\mathbf{0 . 3 8}$ & $\mathbf{0 . 4 3}$ \\
7. T3 Dep & $2.04(0.59)$ & - & - & - & - & - & 1 & $\mathbf{0 . 7 6}$ & $\mathbf{0 . 6 2}$ \\
8. T3 NA & $3.26(1.22)$ & - & - & - & - & - & - & 1 & $\mathbf{0 . 6 6}$ \\
9. T3 NF & $3.56(1.26)$ & - & - & - & - & - & - & - & 1 \\
\hline
\end{tabular}

Bolded terms represent $\mathrm{p}<0.05$

Dep depressive symptoms, $N A$ negative affect, $N F$ need frustration

\begin{tabular}{|c|c|c|c|}
\hline Path & $\mathrm{B}[95 \% \mathrm{CI}], p$ & Path & $\mathrm{B}[95 \% \mathrm{CI}], p$ \\
\hline $\mathrm{T} 1 \mathrm{NA} \rightarrow \mathrm{T} 2 \mathrm{NA}$ & $0.19[0.05,0.34], p=0.007$ & $\mathrm{~T} 1 \mathrm{DEP} \rightarrow \mathrm{T} 2 \mathrm{NF}$ & $0.20[0.05,0.34], p=0.007$ \\
\hline $\mathrm{T} 2 \mathrm{NA} \rightarrow \mathrm{T} 3 \mathrm{NA}$ & $0.33[0.16,0.49], p<0.001$ & $\mathrm{~T} 1 \mathrm{NF} \rightarrow \mathrm{T} 2 \mathrm{NA}$ & $0.18[0.06,0.29], p=0.002$ \\
\hline $\mathrm{T} 1 \mathrm{DEP} \rightarrow \mathrm{T} 2 \mathrm{DEP}$ & $0.46[0.32,0.59], p<0.001$ & $\mathrm{~T} 1 \mathrm{NF} \rightarrow \mathrm{T} 2 \mathrm{DEP}$ & $0.13[0.03,0.25], p=0.01$ \\
\hline $\mathrm{T} 2 \mathrm{DEP} \rightarrow \mathrm{T} 3 \mathrm{DEP}$ & $0.26[0.09,0.43], p=0.002$ & $\mathrm{~T} 2 \mathrm{NA} \rightarrow \mathrm{T} 3 \mathrm{DEP}$ & $0.10[-0.07,0.27], p=0.25$ \\
\hline $\mathrm{T} 1 \mathrm{NF} \rightarrow \mathrm{T} 2 \mathrm{NF}$ & $0.43[0.32,0.54], p<0.001$ & $\mathrm{~T} 2 \mathrm{NA} \rightarrow \mathrm{T} 3 \mathrm{NF}$ & $0.17[0.01,0.34], p=0.04$ \\
\hline $\mathrm{T} 2 \mathrm{NF} \rightarrow \mathrm{T} 3 \mathrm{NF}$ & $0.32[0.18,0.46], p<0.001$ & $\mathrm{~T} 2 \mathrm{DEP} \rightarrow \mathrm{T} 3 \mathrm{NA}$ & $0.01[-0.17,0.17], p=0.97$ \\
\hline $\mathrm{T} 1 \mathrm{NA} \rightarrow \mathrm{T} 2 \mathrm{DEP}$ & $-0.01[-0.15,0.12], p=0.85$ & $\mathrm{~T} 2 \mathrm{DEP} \rightarrow \mathrm{T} 3 \mathrm{NF}$ & $0.01[-0.16,0.18], p=0.88$ \\
\hline $\mathrm{T} 1 \mathrm{NA} \rightarrow \mathrm{T} 2 \mathrm{NF}$ & $-0.07[-0.22,0.06], p=0.27$ & $\mathrm{~T} 2 \mathrm{NF} \rightarrow \mathrm{T} 3 \mathrm{NA}$ & $0.16[0.02,0.31]$, \\
\hline $\mathrm{T} 1 \mathrm{DEP} \rightarrow \mathrm{T} 2 \mathrm{NA}$ & $0.19[0.05,0.34], p=0.007$ & $\mathrm{~T} 2 \mathrm{NF} \rightarrow \mathrm{T} 3 \mathrm{DEP}$ & $0.17[0.03,0.31], p=0.02$ \\
\hline
\end{tabular}

Bolded values indicate significance. STDYX values reported
Table 2 The coefficients for fully cross-lagged model with depressive symptoms, negative affect and need frustration over the academic year

\section{Results}

\section{Preliminary analysis}

Means, standard deviations and correlations between all variables are included in Table 1 below. $^{2}$ Need frustration, depressive symptoms and negative affect were positively correlated.

\section{Main analysis}

To test whether negative affect, need frustration and depressive symptoms were concomitants or antecedents of one another, a fully cross-lagged path model ${ }^{3}$ was created using MPlus software (Muthen \& Muthen, 2017). Missing data was handled using FIML in MPlus. The model had good fit $\left(\mathrm{BIC}=6543.72, \chi^{2}(9)=36.77, \mathrm{p}<0.001\right.$, $\mathrm{RMSEA}=0.09[0.06,0.12], \mathrm{CFI}=0.979, \mathrm{SRMR}=0.048)$.

\footnotetext{
2 All data is available on OSF.

${ }^{3}$ Supplemental analysis was completed to test whether a latent SEM or random intercept cross-lag panel model worked with this data set. The supplemental analysis can be found on OSF.
}

For a summary of the statistics and a display of the model please see Table 2 and Fig. 1. Each variable was a consistent predictor of itself over time, meaning someone who reported more negative affect in September was more likely to report greater negative affect in December and April, and this was true for all the variables. From the beginning of the year to the end of the year, need frustration was the only consistent predictor of negative affect and depressive symptoms over time. In September students who reported greater need frustration experienced more negative affect in December and additionally, students who reported more need frustration in December experienced even more negative affect and depressive symptoms in April. At the beginning of the year, students who reported depressive symptoms reported more negative affect and need frustration in the middle of the academic year. However, reports of depressive symptoms in December did not predict negative affect or need frustration at the end of the year when controlling for all the other variables. Negative affect at the beginning of the year did not predict depressive symptoms or need frustration in the middle of the year, but negative affect in December predicted greater need frustration at the end of the academic year, suggesting a recursive effect of need frustration. Across all the 


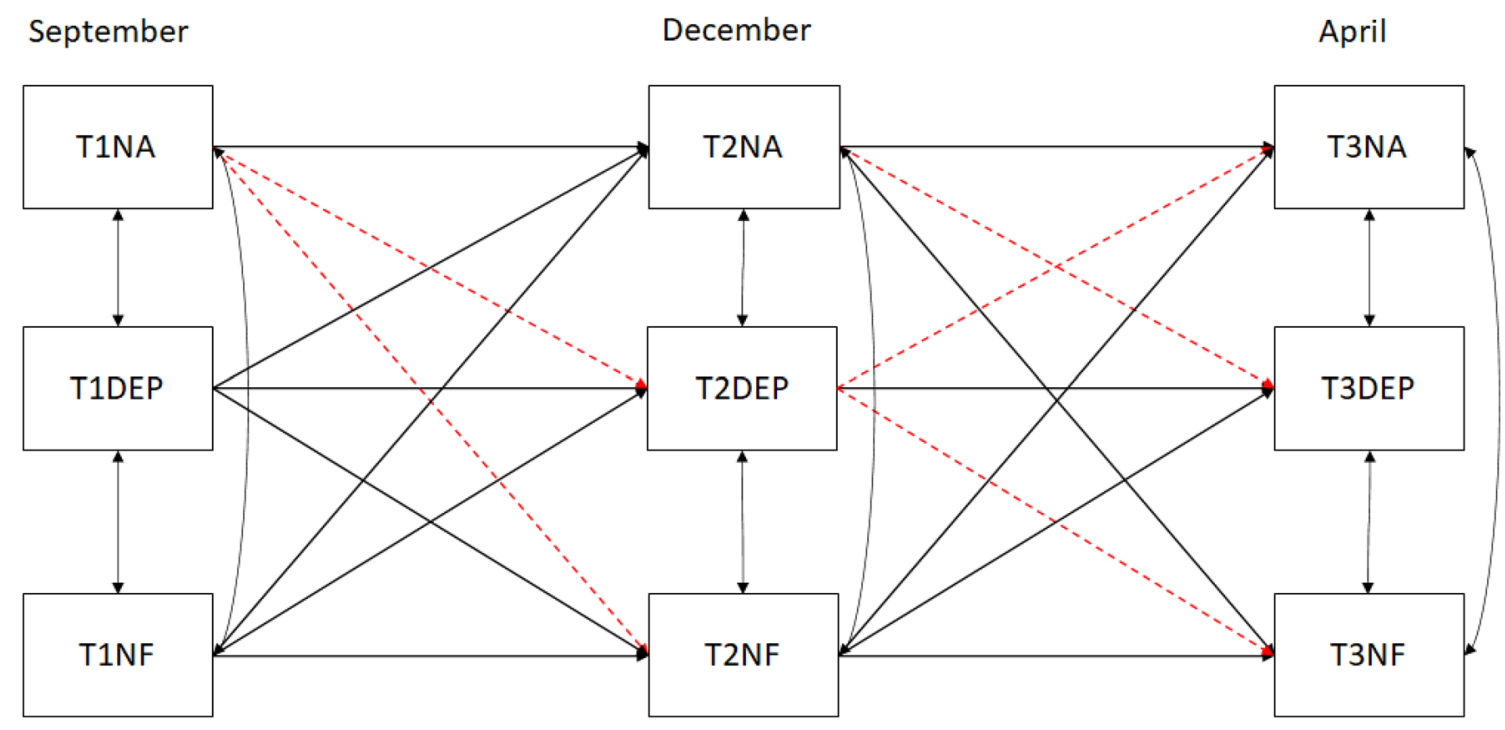

Fig. 1 The fully cross-lagged path model between negative affect, depression and need frustration at the beginning, middle and end of the academic year. Black solid lines represent significant associations, and red dashed lines signify non-significant relations

time points the variables were correlated and this variance was controlled for over time.

\section{Brief discussion}

The current study aimed to distinguish the relative contribution of affect and basic psychological need frustration on the development of depressive symptoms longitudinally. Need frustration was the only consistent predictor of depressive symptoms and negative affect over the academic year. This research provides additional evidence that need frustration is both an antecedent and concomitant for depressive symptoms across the academic year. Meaning it is both correlated over time with depression and anxiety (concomitant) and precedes these outcomes when examined longitudinally (antecedent). Depressive symptoms often increase across the academic year and have become an increasingly prevalent problem across university campuses (Evans et al., 2018; Levine et al., 2021). The current research suggests that need frustration is a risk factor for this decline in mental health.

Depressive symptoms at the beginning of the school year predicted need frustration, and negative affect in the middle of the academic year, but not from the middle of the academic year to the end of the year. Experiencing depressive symptoms is likely to make students feel more need frustrated. For example, a depressed student might withdraw from their social groups (relatedness frustration), be less able to concentrate and study (competence frustration) or feel increasingly overwhelmed with school tasks (autonomy frustration). Again, any combination of these or other events might occur if an individual is feeling more depressed and that might make their environment need frustrating. Students who perceive their environment to be need frustrating are at higher risk of experiencing depressive symptoms, and students who are experiencing depressive symptoms are more likely to find their university environment to be need frustrating.

Negative affect was not found to be associated with later depressive symptoms, but there was a recursive effect, such that mid-year negative affect was related to increased need frustration at the final time point. These findings show that psychological needs, not affect, predict depressive symptoms over the year. However, affect still clearly plays a role in depression, and is highly correlated with depressive symptoms over time. These findings are contrary to previous cross sectional research which has shown that affect does contribute to depressive symptoms (Nima et al., 2013). Our study suggests that negative affect has less incremental validity as a predictor of depressive symptoms when compared to psychological need frustration. This might suggest that one's perception of autonomy, competence and relatedness in their environment is a greater predictor of ill-being, than affect. This study also suggests that need frustration may precede feelings of negative affect. Affective changes associated with depressive symptoms are actually the result of need frustration, which might be why previous studies have shown affect to predict depressive symptoms (Gunnell et al., 2013). Study 2 aims to replicate the current set of novel findings in a sample of university students during the start of the 2020 COVID-19 pandemic. Additionally, this sample uses the reports of close others to examine whether self-reported affect and need frustration predicted the presentation of depressive symptoms. 


\section{Study 2}

The COVID-19 pandemic has been an unprecedented global stressor. During the pandemic, the world experienced adversity together, and a collective uncertainty of what might happen. Preliminary research has found that living through the COVID-19 pandemic was a stressful event and related to increased psychopathology including anxiety, depression, and loneliness (e.g. Cullen et al., 2020; Lei \& Klopack, 2020; Pfefferbaum \& North, 2020). Our emotional and psychological health has been impacted during this time. However, there has yet to be extensive research on how need frustrating this time may have been, and how perceptions of need frustration may have impacted mental health during this time. At the start of the novel COVID-19 pandemic in North America, many students were isolated from others (relatedness frustration), were unable to complete their school year or extracurricular activities normally (competence frustration) and were unable to control or choose what they were able to do (autonomy frustration). Basic psychological need satisfaction has been found to be associated with increased well-being during the COVID-19 pandemic (Cantarero et al., 2020). Additionally, a recent study has found that need frustration predicted increased anxiety and depression over 10 days of lockdown during the pandemic (Vermote et al., 2021).

The goal of this second study was to replicate our previous finding in a pandemic sample. Again, we examined the cross-lagged relations between negative affect, need frustration and depressive symptoms across 6 weeks at the beginning of the global pandemic (early April 2020 to late May 2020). We hypothesized, that need frustration would be an antecedent and concomitant of depressive symptoms and negative affect during the COVID-19 pandemic. A secondary goal of this second study was to examine whether these results could be replicated using the reports of close family members and friends to confirm changes in depressive symptoms associated with need frustration. The reports were intended to corroborate the participant self-reports and help us to further understand depressive presentation in these individuals.

\section{Method}

\section{Participants and procedure}

The 235 participants for this year-long online study were recruited from a large Canadian public university. Participants were university students ( $83 \%$ female, $\mathrm{M}_{\text {age }} 20.82$, $\mathrm{SD}=3.40$ ). The ethnic background of our sample was predominantly European descent (46\%) but included 38\% Asian descent, 3\% African descent, 5\% Middle Eastern descent, and $4 \%$ Hispanic. Recruitment was done through traditional and online platforms such as posters around campus, lecture hall announcements, and Facebook posts. The study was conducted through online surveys administered through Qualtrics at the start of the pandemic in April 2020 (T1), and participants were followed up 6 weeks afterwards in late May 2020 (T2). The surveys assessed psychological needs, affect, and depressive symptoms at each time point. Participants were compensated up to $\$ 20$ for their time. ${ }^{4}$

This study was part of a larger year long research project, and peer and family reports were collected in September 2019 and May 2020. In September, each participant nominated a family member and friend to complete a short survey. The family and friend surveys were brief and asked about the nominating participant's mental health. These reports were entirely voluntary. To maximize these reports, we averaged family and friend reports at each time point. This also allowed us to have a better idea of how the participant was functioning overall. ${ }^{5}$ At the first time point about 200 family members and 204 peers filled out reports, and at the second time point 146 family members, and 144 peers filled out reports (Combined $\mathrm{N}_{\mathrm{T} 1}=242 ; \mathrm{N}_{\mathrm{T} 2}=200$ ). For family member reports, $72 \%$ were parents, $17.5 \%$ were siblings, and the remainder were other relatives. For peer reports, $77 \%$ were friends, $18 \%$ were romantic partners.

\section{Measures}

\section{Affect}

The same measure of affect was used, please see above for detail $\left(\alpha_{\text {NAT } 1}=0.84, \alpha_{\text {NAT2 }}=0.87\right)$.

\section{Basic psychological need frustration}

The same measure of need frustration was used, please see above for detail $\left(\alpha_{\mathrm{NFT} 1}=0.82, \alpha_{\mathrm{NFT} 2}=0.73\right)$.

\section{Depressive symptoms}

The same measure of depressive symptoms was used, please see above for detail $\left(\alpha_{\mathrm{T} 1}=0.84, \alpha_{\mathrm{T} 2}=0.87\right)$.

\section{Depressive presentation}

To measure how depressed a student presented to their family and friends, we used two items from the Big-Five

\footnotetext{
$\overline{4}$ This was a part of a larger year-long goal study. The other measures collected are included on our OSF.

${ }^{5}$ Data for this study is also available on OSF.
} 
Table 3 Mean, standard deviations and correlations between all variables of interest

\begin{tabular}{lllllllll}
\hline Variable & Mean(SD) & 2 & 3 & 4 & 5 & 6 & 7 & 8 \\
\hline 1. T1 Dep & $2.06(0.51)$ & $\mathbf{0 . 7 5}$ & $\mathbf{0 . 6 3}$ & $\mathbf{0 . 6 3}$ & $\mathbf{0 . 5 1}$ & $\mathbf{0 . 4 3}$ & $\mathbf{0 . 2 3}$ & $\mathbf{0 . 3 4}$ \\
2. T1 NA & $3.50(1.08)$ & 1 & $\mathbf{0 . 6 6}$ & $\mathbf{0 . 5 4}$ & $\mathbf{0 . 6 2}$ & $\mathbf{0 . 4 7}$ & $\mathbf{0 . 2 3}$ & $\mathbf{0 . 3 2}$ \\
3. T1 NF & $3.64(1.10)$ & - & 1 & $\mathbf{0 . 5 2}$ & $\mathbf{0 . 5 1}$ & $\mathbf{0 . 6 4}$ & 0.10 & $\mathbf{0 . 2 9}$ \\
4. T2 Dep & $2.29(0.61)$ & - & - & 1 & $\mathbf{0 . 7 7}$ & $\mathbf{0 . 6 6}$ & $\mathbf{0 . 2 8}$ & $\mathbf{0 . 3 7}$ \\
5. T2 NA & 3.95(1.28) & - & - & - & 1 & $\mathbf{0 . 6 3}$ & $\mathbf{0 . 3 1}$ & $\mathbf{0 . 3 7}$ \\
6. T2 NF & 4.02(1.25) & - & - & - & - & 1 & $\mathbf{0 . 1 5}$ & $\mathbf{0 . 2 3}$ \\
7. DepO_0 & 2.34(1.10) & - & - & - & - & - & 1 & $\mathbf{0 . 6 3}$ \\
8. DepO_2 & $2.28(1.11)$ & - & - & - & - & - & - & 1 \\
\hline
\end{tabular}

Bolded terms represent $\mathrm{p}<0.05$.

Dep depressive symptoms, $N A$ negative affect, $N F$ need frustration, Dep $O$ others' reports of depression. DepO_0 is the September 2019 time point, and DepO_2 is the May 2020 measurement
Inventory (BFI; John \& Srivastava, 1999). Reporters rated these items on a 5-point Likert scale ranging from 1 "strongly disagree" to 5 "strongly agree". The items were, "[insert participant's name] is someone who is depressed" and "[insert participant's name] is someone who can be moody". These items were averaged across both supporters to compute a depressive presentation score at each time point $\left(\alpha_{\mathrm{T} 1}=0.62 ; \alpha_{\mathrm{T} 2}=0.67\right)$.

\section{Results}

\section{Preliminary analysis}

Means, standard deviations and correlations between all variables are included in Table 3 below. Need frustration, depressive symptoms and negative affect were positively correlated.

\section{Main analysis}

To test whether negative affect, need frustration and depressive symptoms were concomitants or antecedents of one another, a fully cross-lagged path model was created using MPlus software (Muthen \& Muthen, 2017). Missing data was handled using FIML in MPlus. The model was identified, so fit statistics are not reported. The syntax and output are available on OSF. For a summary of the statistics and a display of the model please see Fig. 2 and Table 4. Each variable was a consistent predictor of itself over the 6-week period. Over this 6 -week period, the only significant crosslagged predictor was need frustration. During the start of the COVID-19 pandemic, individuals who reported finding this experience more need frustrating were more likely to experience negative affect and depressive symptoms six weeks later. Across all the time points the variables were correlated and this variance was controlled for over time.

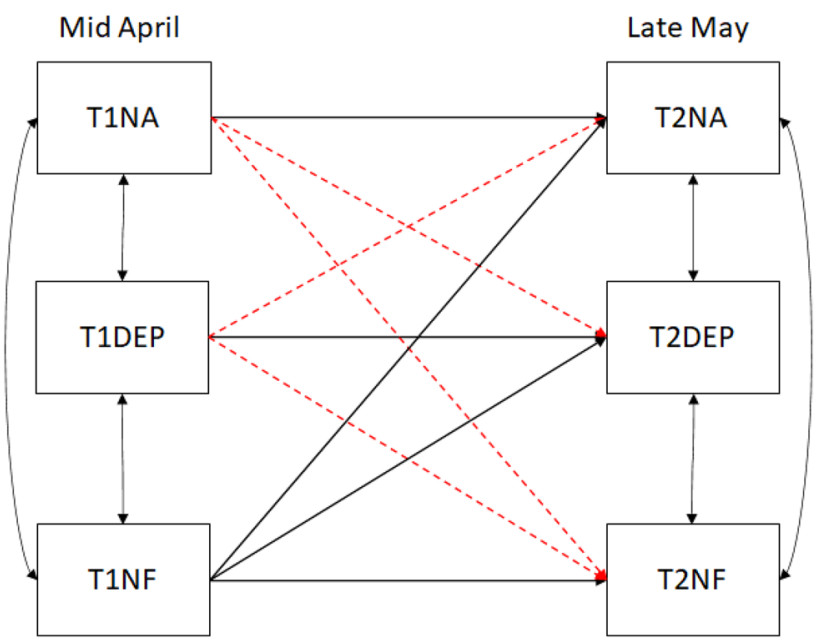

Fig. 2 The fully cross-lagged path model between negative affect, depression and need frustration at the start of the COVID-19 pandemic. Black solid lines represent significant associations, and red dashed lines signify non-significant relations

Table 4 The coefficients for fully cross-lagged model with depressive symptoms, negative affect and need frustration over 6 weeks

\begin{tabular}{ll}
\hline Path & $\mathrm{B}[95 \% \mathrm{CI}], \boldsymbol{p}$ \\
\hline $\mathrm{T} 1 \mathrm{NA} \rightarrow \mathrm{T} 2 \mathrm{NA}$ & $\mathbf{0 . 4 7}[\mathbf{0 . 3 1}, \mathbf{0 . 6 2}], \boldsymbol{p}<\mathbf{0 . 0 0 1}$ \\
$\mathrm{T} 1 \mathrm{DEP} \rightarrow \mathrm{T} 2 \mathrm{DEP}$ & $\mathbf{0 . 4 6}[\mathbf{0 . 3 1}, \mathbf{0 . 6 1}], \boldsymbol{p}<\mathbf{0 . 0 0 1}$ \\
$\mathrm{T} 1 \mathrm{NF} \rightarrow \mathrm{T} 2 \mathrm{NF}$ & $\mathbf{0 . 5 9}[\mathbf{0 . 4 6 , 0 . 7 1}], \boldsymbol{p}<\mathbf{0 . 0 0 1}$ \\
$\mathrm{T} 1 \mathrm{DEP} \rightarrow \mathrm{T} 2 \mathrm{NA}$ & $0.06[-0.10,0.21], p=0.487$ \\
$\mathrm{~T} 1 \mathrm{NF} \rightarrow \mathrm{T} 2 \mathrm{NA}$ & $\mathbf{0 . 1 7}[\mathbf{0 . 0 3 , 0 . 3 0}], \boldsymbol{p}=\mathbf{0 . 0 1 8}$ \\
$\mathrm{T} 1 \mathrm{NA} \rightarrow \mathrm{T} 2 \mathrm{DEP}$ & $0.07[-0.07,0.24], p=0.287$ \\
$\mathrm{~T} 1 \mathrm{NF} \rightarrow \mathrm{T} 2 \mathrm{DEP}$ & $\mathbf{0 . 1 8}[\mathbf{0 . 0 4}, \mathbf{0 . 3 2}], \boldsymbol{p}=\mathbf{0 . 0 1 0}$ \\
$\mathrm{T} 1 \mathrm{NA} \rightarrow \mathrm{T} 2 \mathrm{NF}$ & $0.09[-0.07,0.25], p=0.287$ \\
$\mathrm{~T} 1 \mathrm{DEP} \rightarrow \mathrm{T} 2 \mathrm{NF}$ & $-0.01[-0.16,0.16], p=0.987$ \\
\end{tabular}

Bolded values indicate significance. STDYX values reported 
Table 5 Regression coefficients for participant depression, affect and need frustration predicting others' reports of depression while controlling for others' baseline reports

\begin{tabular}{ll}
\hline Predictor & $\mathrm{B}[95 \% \mathrm{CI}], p$ \\
\hline DepO_0 & $0.62[0.51,0.75], p<0.001$ \\
T1Dep & $0.08[-0.18,0.44] p=0.397$ \\
T1NA & $0.03[-0.13,0.18], p=0.743$ \\
T1NF & $0.14[-0.01,0.29], p=0.074$ \\
\hline
\end{tabular}

DepO_0 is baseline of others' reports of depression

\section{Supplementary analyses: others' reports}

To further confirm our results, we gathered peer and family member reports of depressive symptoms. Using hierarchical linear regression (Table 5), we examined whether participant reports of depressive sympotms, negative affect and need frustration in April at the start of the pandemic would predict others' reports of depression, or dperessive presentation. Baseline reports from others about depressive symptoms, and participant reports of depressive symptoms, affect and need frustration accounted for $48.6 \%$ of the variance in end of year others' reports of depressive symptoms $\left(F(4,165)=39.01, p<0.001, \mathrm{r}^{2}=0.486\right)$. Baseline reports of depressive symptoms predicted end of year reports of depressive sympotms. Need frutration was a marginally significant predictor of others' reports of depressive symptoms when controlling for baseline reports, beta $=0.14, p=0.07$. This suggests that effect of need frustration on depressive symptoms is somewhat observable to close others, thus lending weight to the self-reported effects.

\section{Brief discussion}

This second study replicated our initial results in a pandemic sample, basic psychological need frustration was found to predict depressive symptoms and negative affect during the start of the COVID-19 pandemic. This further supports our initial findings that need frustration is an antecedent of psychological distress. Additionally, these results compliment initial findings that implicate the importance of basic psychological need satisfaction for well-being and ill-being during the pandemic (Cantarero et al., 2020). The novel COVID-19 pandemic was a time of uncertainty and change for many. Individuals who experienced autonomy, competence and relatedness frustration during the pandemic were more likely to experience sadness, loneliness, anger, amotivation, loss of pleasure and overall distress. Additionally, reports from family members and friends confirm this association. Need frustration may represent an important and identifiable predictor of how an individual may cope during a stressful life event, like a pandemic.

\section{Discussion}

Need frustration was found to be an antecedent and concomitant of depressive symptoms over an average academic year, and during the COVID-19 pandemic. Depressive symptoms often increase across the academic year and have become an increasingly prevalent problem across university campuses (Evans et al., 2018; Levine et al., 2021). Need frustration might be one factor that explains who is more likely to experience severe depressive symptoms over time, and during vulnerable periods of time, like the start of a global pandemic.

The current findings further support the assertion that psychological need frustration predicts ill-being (Sheldon \& Gunz, 2009). Not only is need satisfaction central to well-being, but active thwarting of a person's needs is detrimental to one's mental health (Chen et al., 2015). The study of need frustration is still a relatively new area in self-determination theory. The current research further implicates need frustration as a transdiagnostic risk factor, as it was both a predictor of negative affect and depressive symptoms longitudinally. Additionally, this research adds to the literature by examining the impact of need frustration on psychopathology longitudinally over an entire academic year. Students often experience an increase in depressive symptoms over an academic year and this study suggests a new mechanism for understanding how depressive symptoms develop in emerging adulthood (Levine et al., 2021). With the increased prevalence of depression on university campuses, risk factors need to be determined to help identify students who may suffer.

Need frustration is often used as a mediator to explain changes in affect and psychopathology (e.g. Campbell et al., 2018; Costa et al., 2015; Levine, Cherrier, et al., 2020; Levine, Milyavskaya, et al., 2020). The current research provides statistical evidence that need frustration has incremental validity as a predictor of psychopathology over time. The present research is crucial to selfdetermination theory as it provides further evidence for the importance of basic psychological needs as an underlying mechanism of ill-being (Ryan \& Deci, 2017). The current research was a stringent test and suggests that need frustration is an antecedent of depressive symptoms and negative affect over time.

Need frustration also emerged as a marginally significant predictor of depressive symptoms when examining close others' reports. This further strengthens this research by suggesting that an individual's experience of need frustration may also contribute to how much an individual presents as depressed or how noticeable their mental health problems are to others. Alternatively, the peer and family reports remove the problem of shared method variance that 
plagues self-report studies in this area. The easier it is to identify mental health problems, the easier it may be to provide early intervention (Calear \& Christensen, 2010).

\section{Limitations and future directions}

There are limitations to consider within the current research. Although we controlled for depressive symptoms throughout the year, extraneous variables like personality could also be contributing to the observed effect. A study by Nishimura and Suzuki (2016) found that accounting for personality traits attenuated the relationship between psychological need satisfaction and well-being, suggesting the present effects might not be entirely the result of psychological needs. Additionally, our study mainly assessed self-report data from surveys. Although self-report data was corroborated with reports from close family and friends in study 2 , we did not have an expert rating of depressive symptoms. Additionally, this research used only one measure of affect (Emmons, 1992). There is a vast literature on the study of affect, and future research should try to replicate this research with other affect measures. Additionally, we looked at need frustration generally, and future research could examine whether a specific form of need frustration might be most detrimental for mental health problems. Most research examining need frustration does not break down this variable by components (Ryan \& Deci, 2017). Additionally, the current samples were underpowered to run a full latent SEM, so future research should aim to replicate these findings using these more sophisticated analyses. The current study was also observing change overtime and cannot be used as evidence for causation.

Furthermore, the study focused on a specific sample, university students. Further research would be needed to determine whether these findings extend beyond the university setting. However, mental health problems are becoming increasingly prevalent across all university campuses and more research is needed to understand why this is occurring (Ketchen Lipson et al, 2015). Students in demanding programs may experience a particularly need thwarting environment, which would take a toll on their mental health. For instance, enthusiastic first year law students tend to report diminished well-being throughout their education due to the autonomy-thwarting environment (Krieger, 2002; Sheldon $\&$ Krieger, 2007). So, university students might be an especially important population to understand for this research question. To evaluate the universality of need thwarting in contributing to the mental health crisis, future research could assess different populations (e.g. older adults, children) cross culturally.

This research suggests that there may be clinical utility in assessing need frustration in at-risk individuals or individuals seeking treatment. Need frustration appears to be an early indicator of depressive symptoms and determining how to target these frustrated needs may help to reduce the severity of a patient's symptom trajectory. Need frustration is a construct that measures how one perceives the environment, so as a clinician it may be possible to work with patients through multiple modalities to either address factors that contribute to need frustrating environments or maladaptive thinking patterns that exacerbate the perception of need frustration. For example, if a patient reported feeling like a failure (competence frustration), a clinician may work with this individual to restructure this thought or consider the evidence for and against it. Alternatively, if a patient reported feeling isolated, or being bullied, it would be possible to work on interpersonal effectiveness skills to help reduce these need frustrating problems (Teixeira et al., 2019). As such, assessing need frustration may help to guide clinical interventions aimed at overcoming individuals' perceptions of isolation, incompetence, or control, which may reduce depressive symptoms over time.

Need frustration was found to be an early warning sign of future depressive symptoms. Using need frustration as an early screening tool for depressive symptoms could help to identify who might be more likely to experience more severe depressive symptoms. Recognizing need frustration early on and improving need support in universities would improve well-being for subclinical, and perhaps even clinical cases. An intervention could be created to promote healthy response to need thwarting, and to equip students with tools to buffer against the frustration of their basic psychological needs. There are many interventions that promote need satisfaction by focusing on increasing autonomy support within one's environment (Cheon et al., 2016; Edmunds et al., 2007). However, lacking need satisfaction is not equivalent to need frustration. More research is needed to determine what interventions are more effective at buffering against psychological need frustration. Perhaps, an intervention focused on coping tools to help buffer against need frustration might be helpful for college students. Some self-care strategies, like reading have been found to reduce need frustration in college students (Levine, Cherrier, et al., 2020). Future research is needed to see whether other selfcare activities, like yoga or baking could also buffer against need frustration when practiced regularly. Future research is needed to understand how basic psychological needs can be intervened upon to promote well-being in at-risk individuals during emerging adulthood.

\section{Conclusion}

The present study found that need frustration was a predictor of depressive symptoms across a "typical" academic year, and during the COVID-19 pandemic. Additionally, there was some evidence that depressive symptoms and negative affect 
lead individuals to experience more need frustration. Need frustration was the only consistent predictor of depressive symptoms and negative affect over the academic year, and during the COVID-19 pandemic, and even when examining close others' reports. The present study supports the importance of psychological needs in ill-being and psychopathology. Future research is needed to examine the applicability of need frustration as a mechanism which explains ill-being and psychopathology, and ultimately, research is needed to examine how need frustration can be intervened upon to reduce psychological distress in emerging adulthood.

Funding This study was funded by The Social Sciences and Humanities Research Council of Canada (435-2014-20463); Fonds de Recherche du Quebec - Sante (2017-SE-196443).

\section{Declarations}

Conflict of interests The authors declare that they have no conflict of interests.

Ethical approval All procedures performed in studies involving human participants were in accordance with the ethical standards of the institutional and/or national research committee and with the 1964 Helsinki declaration and its later amendments or comparable ethical standards.

Informed consent Informed consent was obtained from all individual participants included in the study.

\section{References}

Andresen, E. M., Malmgren, J. A., Carter, W. B., \& Patrick, D. L. (1994). Screening for depression in well older adults: Evaluation of a short form of the CES-D. American Journal of Preventive Medicine, 10, 77-84. https://doi.org/10.1016/s0749-3797(18) 30622-6

Calear, A. L., \& Christensen, H. (2010). Systematic review of schoolbased prevention and early intervention programs for depression. Journal of Adolescence, 33(3), 429-438. https://doi.org/10.1016/j. adolescence.2009.07.004

Campbell, R., Boone, L., Vansteenkiste, M., \& Soenens, B. (2018). Psychological need frustration as a transdiagnostic process in associations of self-critical perfectionism with depressive symptoms and eating pathology. Journal of Clinical Psychology, 74, 1775-1790. https://doi.org/10.1002/jclp.22628

Cantarero, K., van Tilburg, W. A., \& Smoktunowicz, E. (2020). Affirming basic psychological needs promotes mental well-being during the COVID-19 outbreak. Social Psychological and Personality Science, 12, 821-828.

Chen, B., Vansteenkiste, M., Beyers, W., Boone, L., Deci, E. L., Van der Kaap-Deeder, J., Duriez, B., Lens, W., Matos, L., Mouratidis, A., \& Ryan, R. M. (2015). Basic psychological need satisfaction, need frustration, and need strength across four cultures. Motivation and Emotion, 39, 216-236. https://doi.org/10.1007/ s11031-014-9450-1

Chen, L., Wang, L., Qiu, X. H., Yang, X. X., Qiao, Z. X., Yang, Y. J., \& Liang, Y. (2013). Depression among Chinese University Students:
Prevalence and socio-demographic correlates. PLoS ONE, 8, 1-6. https://doi.org/10.1371/journal.pone.0058379

Cheon, S. H., Reeve, J., \& Song, Y. G. (2016). A teacher-focused intervention to decrease PE students' amotivation by increasing need satisfaction and decreasing need frustration. Journal of Sport and Exercise Psychology, 38, 217-235. https://doi.org/10.1123/jsep. 2015-0236

Cordeiro, P., Paixão, P., Lens, W., Lacante, M., \& Sheldon, K. (2016). Factor structure and dimensionality of the balanced measure of psychological needs among Portuguese high school students. Relations to well-being and ill-being. Learning and Individual Differences, 47, 51-60. https://doi.org/10.1016/j.lindif.2015.12. 010

Costa, S., Cuzzocrea, F., Gugliandolo, M. C., \& Larcan, R. (2015). Associations between parental psychological control and autonomy support, and psychological outcomes in adolescents: The mediating role of need satisfaction and need frustration. Child Indicators Research, 9, 1059-1076. https://doi.org/10.1007/ s12187-015-9353-Z

Cullen, W., Gulati, G., \& Kelly, B. D. (2020). Mental health in the COVID-19 pandemic. QJM: an International Journal of Medicine, 113(5), 311-312. https://doi.org/10.1093/qjmed/hcaa110

Diener, E., \& Oishi, S. (2000). Money and happiness: Income and subjective well-being across nations. Culture and Subjective WellBeing, 8, 185-218.

Edmunds, J., Ntoumanis, N., \& Duda, J. L. (2007). Adherence and well-being in overweight and obese patients referred to an exercise on prescription scheme: A self-determination theory perspective. Psychology of Sport and Exercise, 8, 722-740. https://doi.org/10. 1016/j.psychsport.2006.07.006

Emmons, R. A. (1992). Abstract versus concrete goals: Personal striving level, physical illness, and psychological well-being. Journal of Personality and Social Psychology, 62, 292-300. https://doi. org/10.1037/0022-3514.62.2.292

Evans, T. M., Bira, L., Gastelum, J. B., Weiss, L. T., \& Vanderford, N. L. (2018). Evidence for a mental health crisis in graduate education. Nature Biotechnology, 36, 282-284. https://doi.org/10.1038/ nbt. 4089

Gunnell, K. E., Crocker, P. R. E., Wilson, P. M., Mack, D. E., \& Zumbo, B. D. (2013). Psychological need satisfaction and thwarting: A test of basic psychological needs theory in physical activity contexts. Psychology of Sport and Exercise, 14, 599-607. https:// doi.org/10.1016/j.psychsport.2013.03.007

Jackson, A., Cavanagh, J., \& Scott, J. (2003). A systematic review of manic and depressive prodromes. Journal of Affective Disorders, 74, 209-217. https://doi.org/10.1016/S0165-0327(02)00266-5

John, O. P., \& Srivastava, S. (1999). The Big-Five trait taxonomy: History, measurement, and theoretical perspectives (Vol. 2, pp. 102-138). University of California.

Ketchen Lipson, S., Gaddis, S. M., Heinze, J., Beck, K., \& Eisenberg, D. (2015). Variations in student mental health and treatment utilization across US colleges and universities. Journal of American College Health, 63, 388-396. https://doi.org/10.1080/07448481. 2015.1040411

Krieger, L. S. (2002). Institutional denial about the dark side of law school, and fresh empirical guidance for constructively breaking the silence. Journal of Legal Education, 52, 112-129.

Lei, M. K., \& Klopack, E. T. (2020). Social and psychological consequences of the COVID-19 outbreak: The experiences of Taiwan and Hong Kong. Psychological Trauma: Theory, Research, Practice, and Policy, 12(S1), S35. https://doi.org/10.1037/tra0000633

Levine, S. L., Cherrier, S., Holding, A. C., \& Koestner, R. (2020). For the love of reading: Recreational reading reduces psychological distress in college students and autonomous motivation is the 
key. Journal of American College Health. https://doi.org/10.1080/ 07448481.2020 .1728280

Levine, S. L., Milyavskaya, M., \& Zuroff, D. C. (2020). Perfectionism in the transition to university: Comparing diathesis-stress and downward spiral models of depressive symptoms. Clinical Psychological Science, 8, 52-64. https://doi.org/10.1177/21677 02619865966

Levine, S. L., Tabri, N., \& Milyavskaya, M. (2021). Trajectories of depression and anxiety symptoms over time in the transition to university: Their co-occurrence and the role of self-critical perfectionism. Development \& Psychopathology. https://doi.org/10. 31234/osf.io/zxg8h

Munezero, M., Montero, C. S., Sutinen, E., \& Pajunen, J. (2014). Are they different? affect, feeling, emotion, sentiment, and opinion detection in text. IEEE Transactions on Affective Computing, 5, 101-111. https://doi.org/10.1109/TAFFC.2014.2317187

Muthén, B., \& Muthén, L. (2017). Mplus (pp. 507-518). Chapman and Hall/CRC.

Nima, A. A., Rosenberg, P., Archer, T., \& Garcia, D. (2013). Anxiety, affect, self-esteem, and stress: Mediation and moderation effects on depression. PLOS ONE. https://doi.org/10.1371/journal.pone. 0073265

Nishimura, T., \& Suzuki, T. (2016). Basic psychological need satisfaction and frustration in Japan: Controlling for the big five personality traits. Japanese Psychological Research, 58, 320-331. https:// doi.org/10.1111/jpr.12131

Pfefferbaum, B., \& North, C. S. (2020). Mental health and the Covid19 pandemic. New England Journal of Medicine, 383(6), 510 512. https://doi.org/10.1056/NEJMp2008017

Porter, S. (2018). A descriptive study of post-secondary student mental health crises. College Quarterly, 21, 3.

Raes, F., Smets, J., Nelis, S., \& Schoofs, H. (2012). Dampening of positive affect prospectively predicts depressive symptoms in nonclinical samples. Cognition and Emotion, 26, 75-82. https://doi. org/10.1080/02699931.2011.555474

Rosenthal, B. S., \& Schreiner, A. C. (2000). Prevalence of psychological symptoms among undergraduate students in an ethnically diverse urban public college. Journal of the American College Health Association, 49, 12-18. https://doi.org/10.1080/07448 480009596277

Ryan, R. M., \& Deci, E. L. (2017). Self-Determination Theory: Basic psychological needs in motivation, development, and wellness. Guilford.

Schultz, P. P., Ryan, R. M., Niemiec, C. P., Legate, N., \& Williams, G. C. (2015). Mindfulness, work climate, and psychological need satisfaction in employee well-being. Mindfulness, 6, 971-985. https://doi.org/10.1007/s12671-014-0338-7

Sheldon, K. M., \& Gunz, A. (2009). Psychological needs as basic motives, not just experiential requirements. Journal of Personality, 77, 1467-1492. https://doi.org/10.1111/j.1467-6494.2009. 00589.x

Sheldon, K. M., \& Hilpert, J. C. (2012). The balanced measure of psychological needs (BMPN) scale: An alternative domain general measure of need satisfaction. Motivation and Emotion, 36, 439-451. https://doi.org/10.1007/s11031-012-9279-4

Sheldon, K. M., \& Krieger, L. S. (2007). Understanding the negative effects of legal education on law students: A longitudinal test of self-determination theory. Personality and Social Psychology Bulletin, 33, 883-897. https://doi.org/10.1177/0146167207301014

Song, Y., Huang, Y., Liu, D., Kwan, J. S. H., Zhang, F., Sham, P. C., \& Tang, S. W. (2008). Depression in college: Depressive symptoms and personality factors in Beijing and Hong Kong college freshmen. Comprehensive Psychiatry, 49, 496-502. https://doi.org/10. 1016/j.comppsych.2008.02.005

Storrie, K., Ahern, K., \& Tuckett, A. (2010). A systematic review: Students with mental health problems: A growing problem. International Journal of Nursing Practice, 16, 1-6. https://doi.org/10. 1111/j.1440-172X.2009.01813.x

Teixeira, P. J., Marques, M. M., Silva, M. N., Brunet, J., Duda, J., Haerens, L., \& Hagger, M. S. (2019). Classification of techniques used in self-determination theory-based interventions in health contexts: An expert consensus study. PsyArXiv. https://doi.org/ 10.31234/osf.io/z9wqu

Trivedi, M. H. (2004). The link between depression and physical symptoms. Primary Care Companion to the Journal of Clinical Psychiatry, 6, 12-16. PMID: 16001092.

Vansteenkiste, M., \& Ryan, R. M. (2013). On psychological growth and vulnerability: Basic psychological need satisfaction and need frustration as a unifying principle. Journal of Psychotherapy Integration, 23, 263-280. https://doi.org/10.1037/a0032359

Vansteenkiste, M., Ryan, R. M., \& Soenens, B. (2020). Basic psychological need theory: Advancements, critical themes, and future directions. Motivation \& Emotion, 44, 1-31. https://doi.org/10. 1007/s11031-019-09818-1

Vermote, B., Waterschoot, J., Morbée, S., Van der Kaap-Deeder, J., Schrooyen, C., Soenens, B., Ryan, R., \& Vansteenkiste, M. (2021). Do psychological needs play a role in times of uncertainty? Associations with well-being during the COVID-19 crisis. Journal of Happiness Studies. https://doi.org/10.1007/s10902-021-00398-x

Watson, D., \& Clark, L. A. (1995). Depression and the melancholic temperament. European Journal of Personality, 9, 351-366. https://doi.org/10.1002/per.2410090505

Watson, D., Clark, L. A., \& Carey, G. (1988). Positive and negative affectivity and their relation to anxiety and depressive disorders. Journal of Abnormal Psychology, 97, 346-353. https://doi.org/10. 1037/0021-843X.97.3.346

Wells, V. E., Klerman, G. L., \& Deykin, E. Y. (1987). The prevalence of depressive symptoms in college students. Social Psychiatry, 22, 20-28. https://doi.org/10.1007/BF00583616

Werner-Seidler, A., Banks, R., Dunn, B. D., \& Moulds, M. L. (2013). An investigation of the relationship between positive affect regulation and depression. Behaviour Research and Therapy, 51, 46-56. https://doi.org/10.1016/j.brat.2012.11.001

Zivin, K., Eisenberg, D., Gollust, S. E., \& Golberstein, E. (2009). Persistence of mental health problems and needs in a college student population. Journal of Affective Disorders, 117, 180-185. https:// doi.org/10.1016/j.jad.2009.01.001

Publisher's Note Springer Nature remains neutral with regard to jurisdictional claims in published maps and institutional affiliations. 